Ann. Génét. Sél. anim., 1975, 7 (3), 263-276.

\title{
GÉNÉTIQUE DE LA COULEUR DE LA TOISON DES RACES OVINES FRANÇAISES SOLOGNOTE, BIZET ET BERRICHONNE
}

\author{
J.-J. LAUVERGNE \\ avec la collaboration technique de P. RAFFIER* \\ Département de Génétique animale, \\ Centre national de Recherches zootechniques, I. N. R.A., \\ 78350 Jouy en Josas (France) \\ * Professeur technique adjoint, \\ Lycée agricole de Clermont-Ferrand-Marmilhat, \\ 63370 Lempdes (France)
}

\section{RÉSUMÉ}

L'analyse de croisements réalisés entre les trois races ovines françaises Berrichonne (blanche), Bizet (toute noire à la naissance avec une liste blanche en tête) et Solognote (toute rouge à la naissance) a tout d'abord montré que les dessins blancs observés, bien que parfois assez différents, étaient dus à l'action d'un seul gène. Ce mutant donne sur fond noir le dessin dit points blancs (liste blanche en tête, extrémités des pattes et de la queue blanches) alors que, sur fond rouge, il donne une panachure irrégulière avec assez de blanc. Ce gène est, en outre, dominant à pénétrance incomplète (50 p. roo) chez l'hétérozygote sur fond noir alors que, sur fond rouge, il donne à l'état hétérozygote la panachure irrégulière (avec une pénétrance totale ou subtotale) et une coloration presque toujours totalement blanche chez l'homozygote. Ce comportement particulier du mutant que nous avons appelé $S^{b}(b$ pour Bizet) n'avait, à notre connaissance, pas encore été mis en évidence explicitement. Ce mutant existe avec le même comportement dans d'autres races comme l'Islandais.

Avec l'utilisation en croisements sur le Bizet et le Solognot d'un bélier Finnois présentant le phénotype mouflon noir (noir à ventre blanc) on a pu montrer chez le Mouton l'existence probable de deux séries alléliques capables de contrôler la coloration proprement dite (c'està-dire la répartition des deux pigments mélaniques : noir ou rouge).

- La série d'Extension, qui comporte deux allèles dont l'un $E^{d}$ est dominant sur l'allèle normal $E^{+}$et entraîne épistatiquement la généralisation du noir sur tout le corps. Le Bizet est homozygote pour cet allèle.

- La série Agouti, dont l'action n'est décelable qu'en présence de l'allèle récessif de la série d'Extension à l'état homozygote $\left(E^{+} E^{+}\right)$. On y distingue trois termes: $A^{w h}$ : rouge ; $A^{w}$ : mouflon noir ; $a$ : noir.

La série d'Extension avait déjà été pressentie par certains auteurs mais des preuves définitives de son existence manquaient encore. La série Agouti avait été déjà, quant à elle, bien caractérisée, chez le mouton Islandais en particulier.

Les formules des trois races françaises étudiées pourraient alors s'écrire :

$$
\begin{aligned}
& E^{+} E^{+} A^{w h} A^{w h} S^{b} S^{b} \text { pour le Berrichon } \\
& E^{+} E^{+} A^{w h} A^{w h} S^{+} S^{+} \text {pour le Solognot } \\
& E^{d} E^{d} A^{w h} A^{w h} S^{b} S^{b} \text { pour le Bizet }
\end{aligned}
$$

La formule du bélier Finnois étant $E+E+A^{w} a S^{b} S^{b}$. 
En admettant qu'un certain nombre de races blanches de moutons sont de formule $E+E+A^{w h_{-}}$ $A{ }^{w h} S^{b} S^{b}$ (comme le Berrichon) on explique facilement que le noir ait pu être considéré comme récessif, non pas d'un autre mutant de pigmentation, mais du blanc. Il suffit, en effet ( $A^{\text {wh }}$ étant dominant sur tous les autres termes de la série $A$ gouti) que quelques gènes récessifs $a$ existent dans la race blanche en question. Dès qu'ils se rencontrent pour donner un homozygote $a a$ le gène de panachure change de comportement et révèle le noir avec, au plus, une liste blanche. Au contraire, dans des croisements avec des races noires à points blancs portant le noir dominant $\left(E^{d} E^{d}, A ? A^{?} S^{b} S^{b}\right)$ le blanc se comportera apparemment comme un récessif par rapport au noir par le jeu de l'épistasie de $E^{d}$ sur tous les termes de la série $A$ gouti, y compris le rouge $A^{w h}$.

\section{INTRODUCTION}

Après avoir abordé l'étude de la couleur noire dans la race Bleu du Main (LAUVERGNE, I96I, 62), nous nous sommes intéressé aux différences pour les gènes de coloration entre les 2 races ovines Berrichonne et Solognote (LAUVERGNE, I969).

Nous proposons maintenant une interprétation génétique plus complète des colorations de la toison des deux dernières races citées et de la race Bizet.

\section{MATÉRIEL ETT MÉTHODES}

Les trois races françaises étudiées ont des génotypes colorés bien connus et bien fixés : le Solognot naît entièrement rouge, le Bizet naît tout noir avec une liste en tête, le bout de la queue et des pattes blanc, le Berrichon naît tout blanc et reste blanc. Chez le Solognot et le Bizet on observe, en outre, avec l'âge une canitie progressive de la toison laineuse cependant que les extrémités couvertes de poils gardent leur couleur originale et que quelques poils colorés se maintiennent dans la toison (cf. Quirrer, r965). Les aires de dominance de ces races en France sont indiquées sur la figure $I$.

Les croisements ont été, pour l'essentiel, réalisés sur la troupe expérimentale du Lycée agricole de Marmilhat à Lempdes, près de Clermont-Ferrand (P.-de-D.), à laquelle on a adjoint quelques animaux acquis par le Département de Génétique animale de l'I. N.R.A. (2 béliers et une dizaine de brebis).

Au départ, en 1965, le plan de croisement ne comprenait que les deux races Berrichonne et Bizet. Ce plan de croisement s'est élargi après qu'en I968 on ait acquis un bélier Finnois de phénotype noir à ventre blanc (dit mouflon noir), déterminé, selon toute vraisemblance, par un allèle de la série A gouti (Rendel, I957; Adalsteinsson, I96o ; Searle, 1968). Cet animal pouvait, par conséquent, nous servir de marqueur sûr pour ce locus. Dès le printemps r 969 ce bélier était accouplé à des brebis Bizet à Marmilhat et, en automne de la même année, à des agnelles Solognotes récemment acquises et maintenues au C. N. R. Z., à Jouy en Josas.

En février 1972, le petit troupeau de Jouy était transféré à Marmilhat où un bélier Solognot était à son tour introduit au printemps 1973, afin de faire les comparaisons entre Bizet et Solognot.

Dans son complet développement le plan de croisement, encore en progression, s'articule autour de deux thèmes :

- accouplement entre les trois races françaises $\left(F_{1}\right.$ et retours),

- accouplement entre le bélier Finnois et les deux races françaises pigmentées Solognote et Bizet $\left(\mathrm{F}_{1}\right.$, retours et croisement rotatif).

Les croisements de retour ont été faits sur le parent récessif en cas de dominance complète et sur les deux parents lorsque les $F_{1}$ étaient intermédiaires. Le croisement dit rotatif a consisté à accoupler une $\mathrm{F}_{1}$ Finnois $\times$ Bizet à des Solognots.

Ajoutons que, pour une partie des données de croisements entre Berrichons et Solognots, on a fait appel à des résultats observés dans les troupeaux de M. de la SELlE (Bièvre) et de l'I. N. R. A. (La Sapinière près de Bourges) et déjà utilisés dans une précédente étude, LAUVERGNE (1969). Cette analyse portait alors, sur une seule différence allélique à un locus de panachure. 


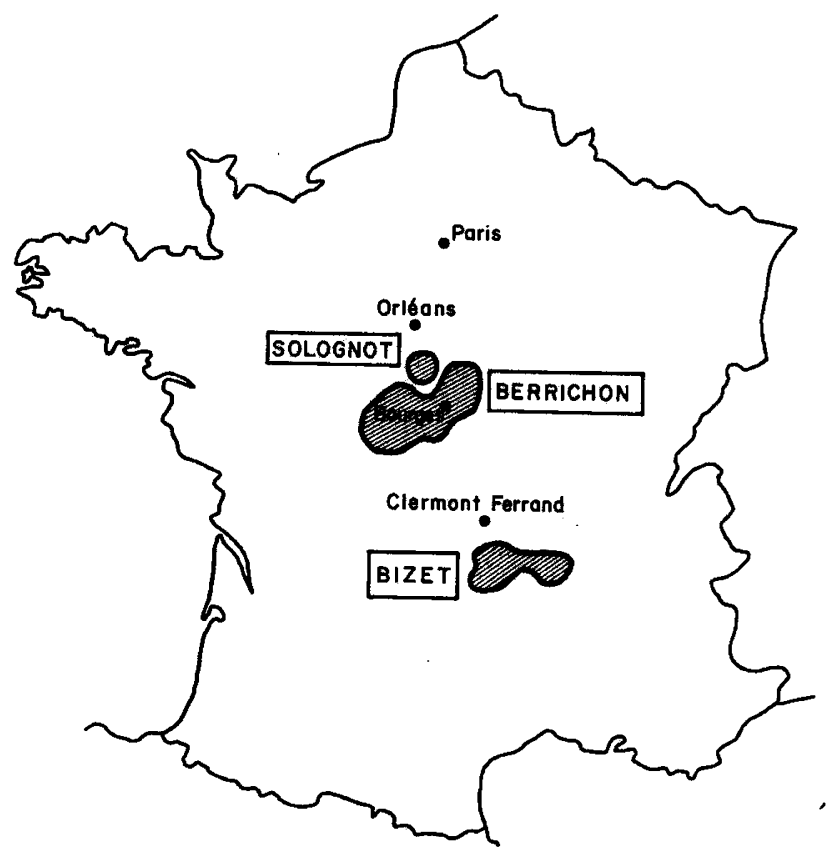

FI(i. I. -- Aire de dominance en France des trois races Solognote, Berrichonne et Bizet (d'après QuitTET, I965)

\section{RÉSULTATS ET CROISEMENTS}

\section{I. - Les phénotypes observés}

Dans les divers croisements de nouveaux phénotypes colorés sont apparus. On donne leur nomenclature et leur description ainsi que celles des phénotypes des races parentales dans le tableau $\mathrm{I}$.

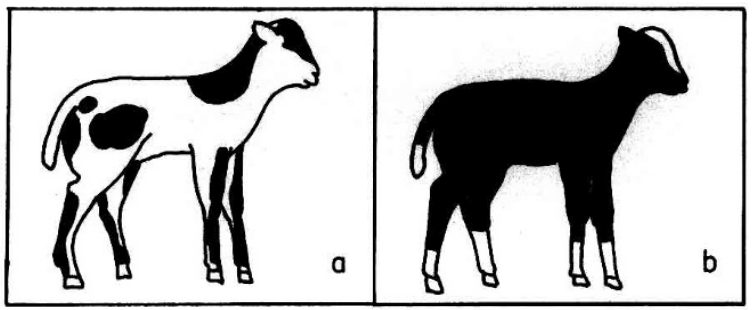

FIG. 2. - Les deux types de dessin blanc chez le Mouton

a) La panachure irrégulière (animaux dits pies) : les plages blanches ont une extension variable sur tout le corps. Dans les types les plus blancs la couleur a tendance à se réfugier aux extrémités. Ce dessin s'observe sur fond rouge dans divers croisements.

b) Les points blancs: Le corps reste coloré, les marques blanches se limitent à une liste en tête, au bout de la queue et des pattes. Ce dessin s'observe sur fond noir ou mouflon noir, il est, en particulier, caractéristique de la race Bizet. 
Phénotypes observés à la naissance dans les expériences de croisement

\begin{tabular}{|c|c|c|}
\hline $\begin{array}{l}\text { Nomenclature } \\
\text { du phénotype }\end{array}$ & Description du phénotype & Races ou croisements ou le phénotype existe \\
\hline noir à points blancs & $\begin{array}{l}\text { noir avec liste blanche en tête, } \\
\text { socquettes blanches et bout de } \\
\text { queue blanche }\left({ }^{1}\right)\end{array}$ & $\begin{aligned}- & \text { le } \text { Bizet } \\
- & \text { les } \mathrm{F}_{1} \text { Berrichon } \times \text { Bizet } \\
- & \text { certains } \mathrm{F}_{1} \text { Solognot } \times \text { Bizet } \\
- & \text { les } \mathrm{F}_{1} \text { Finnois } \times \text { Bizet } \\
- & \text { certains back-cross Berrichon } \times(\text { Berrichon } \times \\
& \text { Bizet }) \\
- & \text { certains back-cross Finnois } \times(\text { Finnois } \times \\
& \text { Bizet })\end{aligned}$ \\
\hline noir & tout noir & $\begin{array}{l}- \text { certains } \mathrm{F}_{1} \text { Solognot } \times \text { Bizet } \\
- \text { certains back-cross Solognot } \times(\text { Berrichon } \times \\
\quad \text { Solognot }) \\
- \text { certains back-cross Solognot } \times(\text { Solognot } \times \\
\quad \text { Bizet }) \\
- \text { certains Solognot } \times(\text { Finnois } \times \text { Bizet }) \\
- \text { certains back-cross Finnois } \times(\text { Finnois } \times \\
\quad \text { Solognot })\end{array}$ \\
\hline $\begin{array}{l}\text { moufton noir }\left({ }^{2}\right) \\
\text { à points blancs }\end{array}$ & $\begin{array}{l}\text { dos noir, ventre régulièrement } \\
\text { blanc avec, parfois, des traces } \\
\text { de rouges, marques blanches } \\
\text { ou rouges symétriques sur la } \\
\text { face; liste blanche en tête, } \\
\text { socquettes et bout de la queue } \\
\text { blanches (1) }\end{array}$ & $\begin{array}{l}\text { - le bélier Finnois } \\
\text { - certains back-cross Bizet } \times(\text { Finnois } \times \text { Bizet }) \\
\text { - certains back-cross Finnois } \times(\text { Finnois } \times \text { So- } \\
\quad \text { lognot })\end{array}$ \\
\hline rouge & tout rouge & $\begin{array}{l}\text { - Le Solognot } \\
\text { - certains back-cross Berrichon } \times \text { (Berrichon } \\
\quad \times \text { Solognot) } \\
\text { - certains back-cross Solognot } \times(\text { Finnois } \\
\quad \times \text { Solognot })\end{array}$ \\
\hline pie rouge & $\begin{array}{l}\text { dessin blanc irrégulier sur tout le } \\
\text { corps }\end{array}$ & $\begin{array}{l}\text { - les } \mathrm{F}_{1} \text { Berrichon } \times \text { Solognot } \\
\text { - les } \mathrm{F}_{1} \text { Finnois } \times \text { Solognot } \\
\text { - certains back-cross Solognot } \times \text { Bizet } \\
\text { - certains Solognot } \times(\text { Finnois } \times \text { Bizet })\end{array}$ \\
\hline blanc & tout blanc & $\begin{array}{l}\text { - le Berrichon } \\
\text { - certains back-cross Berrichon } \times(\text { Berrichon } \\
\quad \times \text { Solognot }) \\
- \text { certains back-cross Solognot } \times(\text { Berrichon } \\
\quad \times \text { Solognot) } \\
- \text { certains back-cross Berrichon } \times(\text { Berrichon } \\
\quad \times \text { Bizet }) \\
- \text { certains back-cross Finnois } \times(\text { Finnois } \times \\
\quad \text { Solognot })\end{array}$ \\
\hline
\end{tabular}

(1) Ces marques blanches en tête, pattes et queue ne sont pas toujours toutes présentes ni toutes bien développées, souvent, au lieu de la liste, on a seulement une étoile en tête.

(2) En français on dit aussi noir et feu (cf. LAUvergne, 1971) le terme mouflon noir est la traduction du terme anglais employé par Berge (1958) et repris par Adalsteinsson (1970). Dans cette langue on dit aussi reversed badger face : face de blaireau inversée, terme qui semble un peu recherché. 

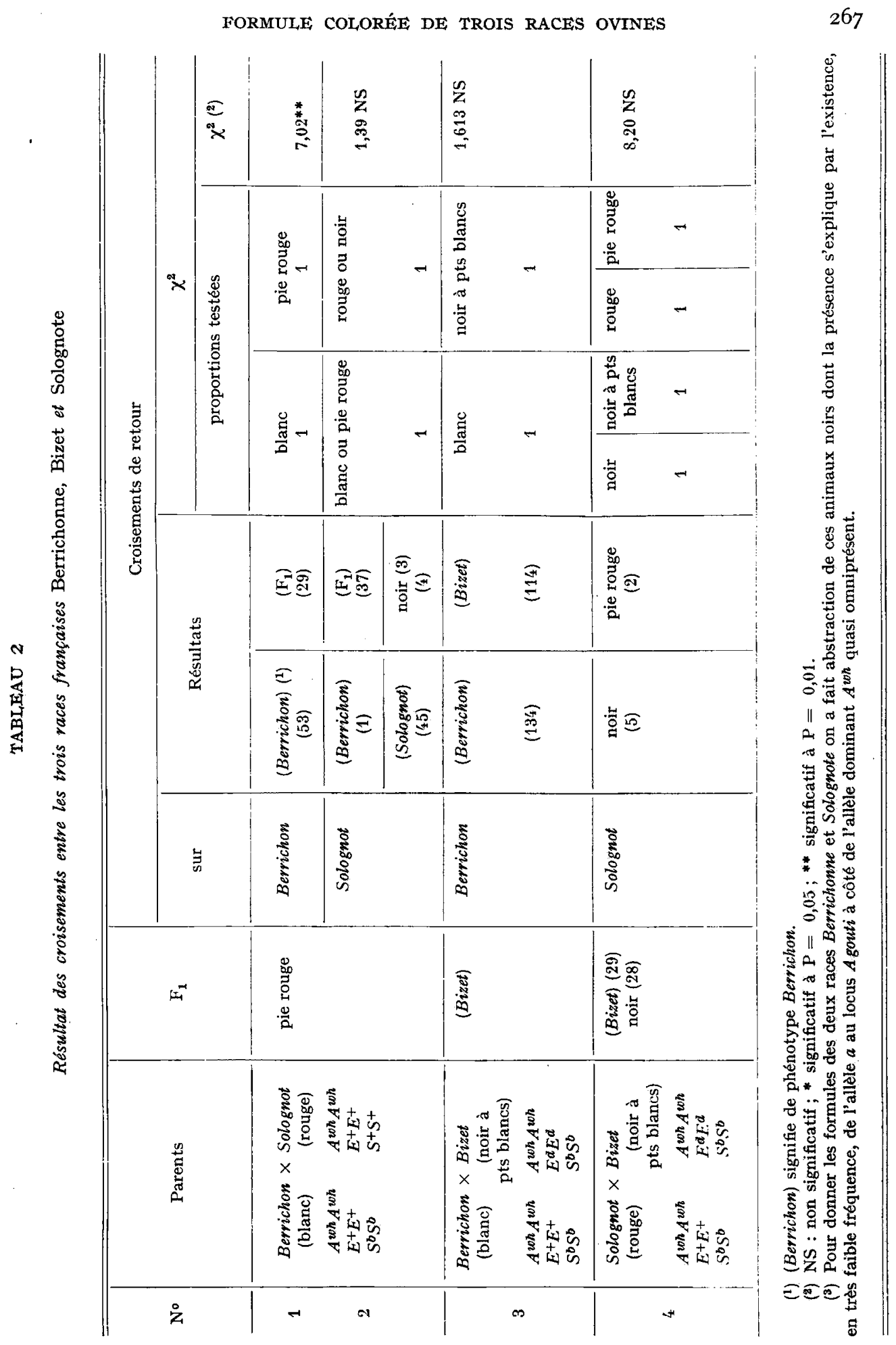


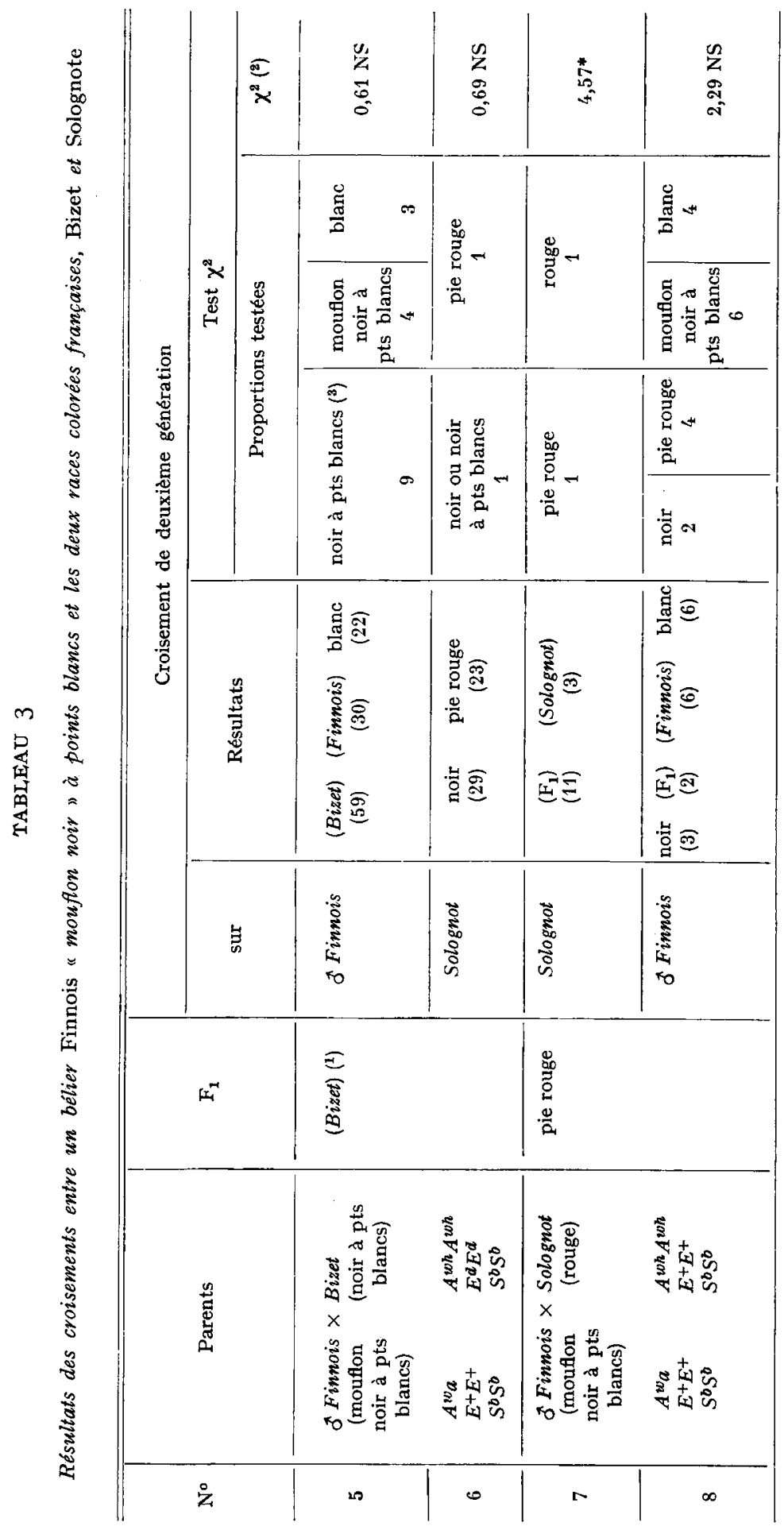


Il s'agit, bien entendu des phénotypes observés à la naissance, on a fait abstraction de la canitie progressive des moutons noirs ou rouges.

Les croquis de la figure 2 permettent de faire la différence entre les deux dessins blancs : le dessin dit "points blancs" et le dessin que nous avons appelé " panachure irrégulière " ou, plus brièvement, pie.

\section{2. - Les ségrégations observées}

Le tableau 2 est consacré aux croisements entre les trois races françaises Berrichonne, Bizet et Solognote.

Le tableau 3 est consacré aux croisements entre les deux races françaises pigmentées. (Bizet et Solognote) et le bélier Finnois.

Dans les tableaux 2 et 3 on donne également les formules géniques des différentes races en présence, telles que nous les déduirons plus loin de notre analyse, ainsi que les $\chi^{2}$ pour tester nos hypothèses sur les ségrégations de deuxième génération.

\section{INTERPRÉTATION MENDÉLIENNE DES SÉGRÉGATIONS OBSERVÉES}

L'analyse successive des croisements entre les trois races françaises (tabl. 2) puis des comparaisons du bélier Finnois aux deux races colorées (tabl. 3) permet de sérier les problèmes :

Tout d'abord, en admettant - comme il semble raisonnable de la faire chez les Mammifères (SEARLE, I968) - que les mutants de dessins blancs ne peuvent être allèles des mutants qui conditionnent la répartition du pigment mélanique noir ou rouge $\left(^{(}\right)$on peut dégager de l'analyse des ségrégations entre Berrichon, Bizet et Solognot l'existence d'un facteur de panachure blanche au comportement original.

Les confrontations entre le bélier Finnois et les deux races pigmentées Bizet et Solognote permettent ensuite de déceler l'existence de deux séries alléliques pour le déterminisme de la pigmentation proprement dite.

\section{I. - Mise en évidence du comportement original d'un facteur pour le dessin blanc, d'après les résultats des croisements entre Berrichon, Bizet et Solognot}

a) Formulation de l'hypothèse.

Tout d'abord, à part 1'apparition en back-cross de la ligne 3 (tabl. 2) de quelques animaux tout noirs que nous expliquerons plus loin, on peut admettrel'existence d'une alternative mendélienne noir/rouge. Les Solognots et les Berrichons étant rouges homozygotes, le Bizet étant noirs homozygotes.

Pour la génétique de la répartition du blanc ensuite que 1'on s'est accordé (cf. plus haut) pour considérer comme distincte de celle de la pigmentation proprement

(1) De toute façon l'hypothèse d'un seul locus ne cadrerait pas avec les résultats du back-cross Solognot par (Solognot $\times$ Bizet) ligne 4 tableau 2 . 
dite on pourrait alors à première vue, penser qu'il y a au moins deux mutants impliqués. L'un donnerait la panachure irrégulière telle que nous l'avons étudiée entre Solognots et Berrichons (LAUVERGNE, I969) l'autre la liste blanche en tête et les extrémités blanches aux pattes et à la queue.

Cette interprétation se heurte toutefois à certaines contradictions, en effet :

- dans la $F_{1}$ Berrichon $\times$ Bizet (ligne 3 du tabl. 2) on n'observe aucune panachure irrégulière en $F_{1}$ alors que l'on a vu qu'en $F_{1}$ Berrichon $\times$ Solognot tous les animaux étaient pies. En outre, en retour sur le Berrichon on n'observe aucun pie rouge mais seulement des blancs ou des noirs à points blancs.

- le croisement de retour de la ligne 4 tableau 2 Solognot $\times($ Solognot $\times$ Bizet $))$ fait apparaître des pies rouges or, le seul gène de dessin blanc introduit est celui de points blancs, porté par le parent Bizet.

On peut lever ces contradictions en admettant que les deux gènes de dessin blanc n'en font, en réalité, qu'un seul, dont l'action serait variable selon le fond coloré :

- sur fond rouge il donnerait, à l'état hétérozygote, de la panachure irrégulière et, à l'état homozygote du blanc (lignes I et 2 tabl. 2),

- sur fond noir il donnerait les points blancs à l'état homozygote et, à l'état hétérozygote, aurait une pénétrance incomplète (estimable à $50 \mathrm{p}$. roo d'après la $\mathrm{F}_{1}$ Bizet $\times$ Solognot de la ligne $4\left({ }^{1}\right)$ ).

\section{b) Vérification de l'hypothèse.}

Les tests $\chi^{2}$ du tableau 2 n'infirment pas notre hypothèse. L'écart constaté à la ligne $\mathrm{r}$ est imputable à des variations d'expressivité déjà analysées (LAUVERGNE, r969) : chez certains hétérozygotes $S^{b} S^{+}$l'extension de la panachure est déjà totale, grâce à l'accumulation de modificateurs.

Cette hypothèse explique assez bien également les résultats observés dans les croisements où intervient le bélier Finnois qui, lui aussi, est à points blancs : deuxièmes générations de la ligne 5 (tabl. 3) où apparaissent des blancs, et des lignes 7 et 8 où apparaissent des pie rouges.

Il s'agit sans doute du même mutant qu'AdALSTEINSSON (I970) a isolé en Islande et qu'il nomme $S_{2}$ puis, plus tard, $s$ (ADALSTEINsson, I974). Retenant le symbole $S$ pour le locus nous proposons l'appellation $S^{+}$pour l'allèle non muté et $S^{b}$ pour 1'allèle de panachure ( $b$ rappelant la race $\left.B i z e t\right)$ : on ne peut en effet guère retenir $s$ qui évoquerait un comportement récessif, ce qui n'est pas toujours vrai.

\section{2. - Interprétation bifactorielle de la génétique de la couleur proprement dite, à partir des croisements où intervient le bélier Finnois}

\section{a) Formulation de l'hypothèse.}

On a vu plus haut qu'il était possible de déceler entre Bizet et Berrichon ou Solognot une alternative mendélienne où un noir uniforme dominerait un rouge uniforme.

(1) On admet que, dans la race Bizet, l'homozygotie totale a été atteinte au locus $S$ pour la formule $S^{b} S^{b}$, vue l'ancienneté de la sélection et l'absence de ségrégation généralement observée. 
Possédant, avec le bélier Finnois mouflon noir, un marquage du locus Agouti on peut tester maintenant si cette alternative mendélienne se situe à ce locus.

Dans l'hypothèse d'une seule série allélique les phénotypes des $\mathrm{F}_{1}$ Finnois $\times$ Bizet et Finnois $\times$ Solognot des lignes 5 et 7 du tableau 3 montrent que 1 'on doit avoir les rapports de dominance suivants : noir $>$ rouge $>$ mouflon noir. Or :

- dans les retours Finnois $\times$ (Finnois $\times$ Bizet) de la ligne 5, tableau 3, il apparait des animaux tout blancs, donc porteurs du facteur pour le rouge (cf. plus haut) alors que le Finnois ne peut porter le facteur rouge et que le Bizet ne le porte pas, d'après la $F_{1}$ Solognot $\times$ Bizet de la ligne 4 tableau 2 .

- dans les retours Finnois $\times$ (Finnois $\times$ Solognot) de la ligne 8 (tabl. 3) apparaissent des noirs alors que ni le Finnois, ni le Solognot ne peuvent porter le noir.

Dans ces conditions il semble bien difficile de ranger tous les mutants de pigmentation dans une seule série allélique. Les faits que nous observons s'expliquent beaucoup plus aisément en postulant, à côté de la série Agouti, une série dite d'Extension telle que la préconisait déjà RENDEL (I957), en raisonnant par homologie avec les rongeurs.

La série Agouti comprendrait les trois termes, par ordre de dominance: rouge > mouflon noir $>$ noir : $A^{w h}, A^{w}, a$ selon la terminologie d'ADALSTEINSSON (I974). Les races Berrichonne et Solognote seraient homozygotes pour $A^{w h}$.

$\mathrm{La}$ série d'Extension comprendrait les deux termes $\mathrm{E}^{a}>\mathrm{E}^{+}, \mathrm{E}^{d}$ qui donne l'extension uniforme du noir étant, en outre, épistatique sur tous les génotypes en Agouti. Le Bizet serait homozygote pour $E^{d} E^{d}$, les autres races seraient $E^{+} E^{+}$.

\section{b) Test de l'hypothèse.}

Pour tester cette hypothèse de dihybridisme il faut savoir quelle est la formule en $A g o u t i$ du bélier Finnois ( $\left.{ }^{1}\right)$. Ce dernier peut en effet être $A^{w} A^{w}$ ou $A^{w} a$ car $A^{w}$ est dominant sur $a$. Il faut également connaître la formule en Agouti des Bizets où $E^{d}$ masque toutes les manifestations des allèles de ce locus.

- d'après les retours Finnois $\times$ (Bizet $\times$ Finnois) de la ligne 5, tableau 3, les Bizets ne peuvent qu'être homozygotes pour l'allèle rouge en Agouti car il apparaît des blancs dont le fond coloré est obligatoirement rouge (cf. plus haut). La formule colorée du Bizet peut donc s'écrire : $A^{w h} A^{w h} E^{a} E^{d} S^{b} S^{b}$.

- d'après les retours Finnois $\times$ (Finnois $\times$ Solognot) de la ligne 8 , tableau 3 , où apparaissent des tout noirs on peut considérer que le bélier Finnois est hétérozygote mouflon noir et noir. Sa formule colorée est donc : $A^{w} a E^{+} E^{+} S^{b} S^{b}$.

Dans ces conditions, en supposant que le Solognot est $A^{w h} A^{w h} E+E^{+} S^{+} S^{+}$, on obtient en deuxième génération les proportions qui sont testées par le $\chi^{2}$ dans le tableau 3. On constate que les valeurs de ce test ne contredisent nulle part l'hypothèse émise.

Avec cette nouvelle conception on voit en outre que l'apparition de noirs à la ligne $2\left(^{2}\right) \mathrm{du}$ tableau 2 sur laquelle nous ne nous étions pas arrêté précédemment s'explique aisément : certains Solognots et Berrichons sont hétérozygotes au locus Agouti: $A^{\text {wh }}$ a.

(1) Rappelons que c'est le même bélier Finnois qui a été employé dans tous les croisements du tableau 2.

(2) Ces animaux noirs sont apparus seulement dans des croisements entrepris pour compléter les données que nous avions de notre première étude Solognot/Berrichon (LAUvERGNE, I969). 
J.-J. LAUVERGNE

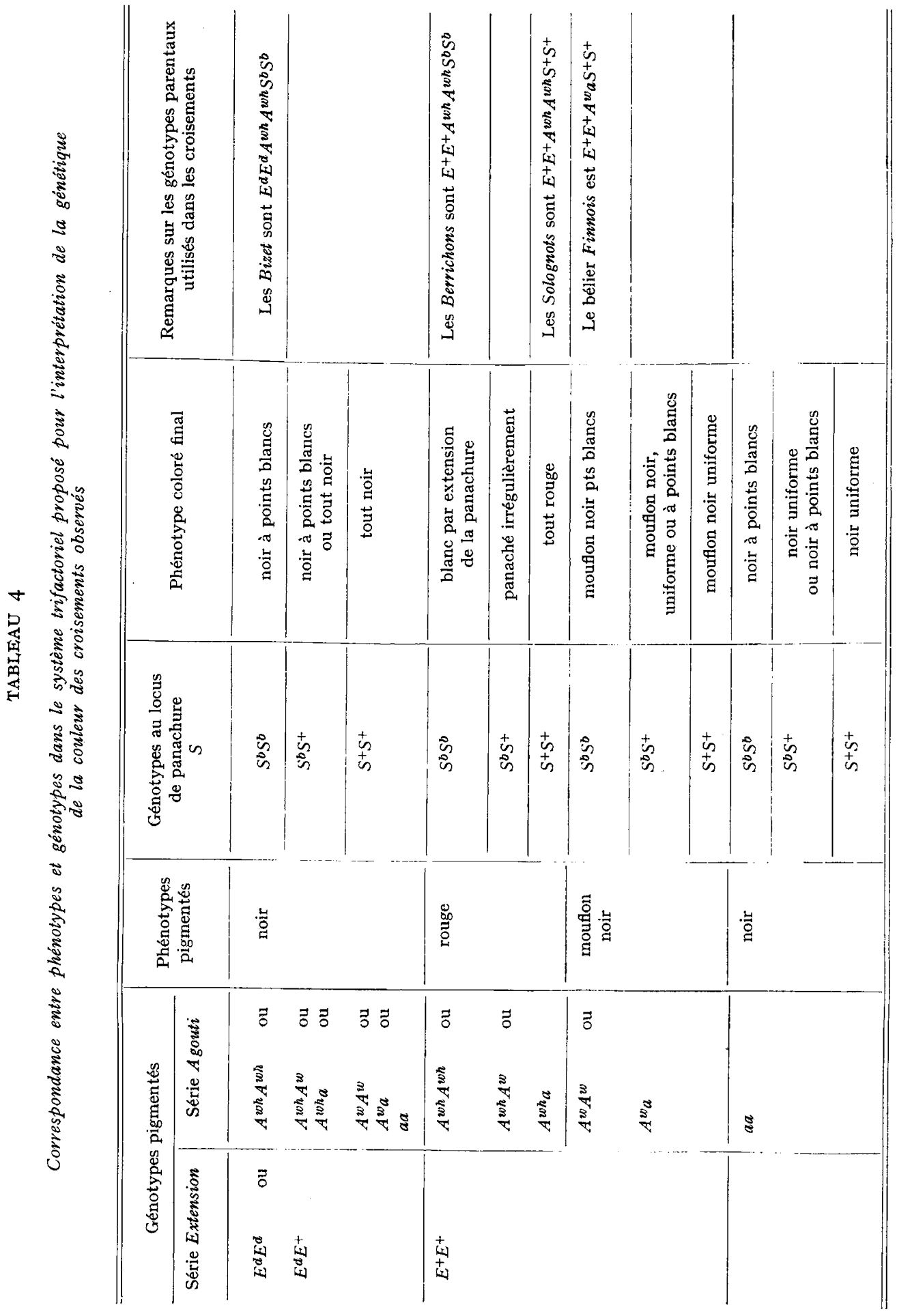




\section{3. -- Le tableau des actions et des interactions des gènes pour la pigmentation et la panachure}

Pour fixer les idées, nous avons, dans le tableau 4, donné le schéma des interactions alléliques et génotypiques qui, selon nous, expliquent les phénotypes colorés observés.

\section{DISCUSSION}

En ce qui concerne la pigmentation ( $\left.{ }^{1}\right)$ nous l'avons vu, nos résultats sont en accord pour la série Agouti avec ceux obtenus déjà par Adalsteinsson (I960) après que RENDEL (I957) ait pressenti chez le mouton l'existence de cette série allélique pour la pigmentation, la plus constamment présente chez tous les Mammifères. SEARLE (I968) raisonne dans le même sens, bien qu'il ignore encore les travaux des deux auteurs déjà cités.

RENDEL (I957) nous l'avons vu plus haut semble aussi être le premier à avoir parlé d'homologie pour la série d'Extension chez le Mouton. Adalsteinsson (I970, I974) lui emboîte le pas et SEARLE (opus cité) raisonne de la même manière.

En fait, l'existence d'un noir dit dominant à côté d'un noir dit récessif était postulé depuis très longtemps en génétique de la coloration du Mouton (cf. RAE, I956). Très souvent toutefois le comportement en dominance du noir se faisait apparemment non pas vis-à-vis d'une couleur pigmentaire mais vis-à-vis du blanc ce qui, en génétique de la couleur des mammifères, est difficilement admissible (cf. SEARLE, cité plus haut).

En montrant que ce comportement n'est qu'un artefact et que, toutes les fois que l'on observe un tout blanc il faut se dire que l'animal en question porte le facteur pour le rouge on a pu aller un peu plus loin qu'un auteur comme HoogschaGEN (I967) qui a déjà établi que les deux noirs (récessif et dominant par rapport au blanc) étaient à des loci différents. On est sûr maintenant que le " noir dominant " est épistatique sur toutes les manifestations d'au moins 3 allèles en $\operatorname{Agouti}\left(A^{w h}, A w, a\right)$ comme le pensait déjà Adalsteinsson (I970).

Pour revenir plus particulièrement sur le gène de dessin blanc, son comportement dans le passé ne paraît pas avoir été explicitement, soupçonné. La solution semble cependant contenue, au moins implicitement, chez Adalsteinsson (1970). En montrant tout d'abord que les tout blancs portent le même facteur en Agouti $\left(A^{w h}\right)$ que les rouges ou les pie rouges, cet auteur nous met tout d'abord incontestablement sur la voie d'une interaction entre un facteur de panachure et un facteur de pigmentation : les animaux à fond noir semblent incapables de devenir tout blancs par extension de la panachure. En outre l'analyse des dessins blancs parmi les moutons Islandais non blancs (ni rouges ni pie rouges) révèle que le dessin le plus courant, et de loin, est le patron "points blancs". Or il n'y a aucune raison de penser - en l'absence de linkage et avec le degré de panmixie pour la couleur qui existe en Islande - que les gènes de panachure différent selon le type de pigmentation mélanique.

(1) Rappelons que le blanc n'est pas une couleur pigmentaire mais le résultat de l'absence de pigments mélaniques. 
Nous devons remarquer, au passage, que, si nous avons pu pousser si loin l'analyse c'est que nous avons eu la chance de disposer : I) d'une race homozygote pour le rouge uniforme, $A^{w h} A^{w h} S^{+} S^{+}:$le Solognot ; 2) d'une race présentant le noir dominant $E^{d} E^{d}$ avec, au locus $A$ gouti, une homozygotie pour $A^{w h} A^{w h}:$ le $B i z e t$; 3) d'un bélier mouflon noir hétérozygote pour le noir récessif : le bélier Finnois. Sans lui la seule manifestation du noir récessif aurait été dans le back-cross de la ligne 2 du tableau 2 ( 4 animaux sur 87 ).

Certaines précisions et confirmations seront apportées dans le futur lorsque tous les accouplements prévus seront réalisés. Il conviendra en particulier de revenir sur l'expressivité du gène $S^{b}$ sur fond noir. Bien que nous n'ayons pas trouvé une variation aussi grande que celle des auteurs australiens comme BroOKER et Doling (I965) ou Hayman et CoOper (I965) (et cela a été, dans une certaine mesure, là encore, une chance pour nous) des écarts au phénotype assez bien fixé du Bizet ont été observés et demandent à être analysés plus précisément.

$$
\text { Reçu pour publication en octobre } 1975 .
$$

\section{REMERCIEMENTS}

Ce travail n'aurait pu voir le jour sans la politique de collaboration scientifique active avec l'I. N. R. A. de la Direction du Lycée Agricole de Clermont-Ferrand-Marmilhat qui, depuis dix ans, a accepté de convertir partiellement sa troupe de brebis en une unité expérimentale pour l'étude de la coloration du Mouton.

Le bélier Finnois mouflon noir né en 1968 de parents blancs récemment importés de Finlande était la propriété de M. JACLOT, ferme de Chantemerle, Le Plessis-Belleville (Oise). Il nous a été gracieusement cédé par cet éleveur après que A. Desvignes (qui travaillait alors au Département de Génétique animale de l'I. N. R. A.) ait vu tout l'intérêt que pouvait avoir un animal présentant un tel phénotype coloré, encore inconnu en France.

Les agnelles Solognotes de l'expérience ont été achetées chez M. de la SELLE, Domaine de Bièvre, Marcilly-en-Gault (Loir-et-Cher) et le bélier de la même race provient de l'élevage de M. P. Pinguet, Granchamps, à Nançay par Neuvy-sur-Barangeon (cher).

Pour l'analyse des données nous remercions le Dr Adalsteinsson (Institut de Recherches agronomiques, Reykjavik, Islande) qui a mis à notre disposition son immense expérience en matière de coloration du mouton.

Nous sommes aussi redevable au Dr Melinda Burricl (Department of Animal Science, University of Minnesota, St Paul, U. S. A.), d'une petite remarque qui donnait la clé de l'analyse du comportement du gène de dessin blanc.

Le Dr Mérat (Génétique animale I. N.R.A.), enfin, a relu en détail le manuscrit et suggéré des corrections fort justifiées.

\section{SUMMARY}

\section{GENETICS OF FLEECE COLORING OF THREE FRENCH SHEEP BREEDS :} SOLOGNOT, BIZET AND BERRICHON

Analyzing crosses between three French sheep breeds Berrichon (white), Bizet (all black at birth with white blaze, tail end and sockings) Solognot (all red at birth) it was first showed that the white patterns studied, although sometimes different, are due to the action of a single gene. On black ground, this mutant gives a pattern called white spots (white blaze, extremities 
of legs and tail), while, on red ground it produces irregular piebaldness with some white. Moreover, this gene is dominant with incomplete penetrance (50 p. I00) it the heterozygote on black ground while, on red ground it gives irregular piebaldness (with total or subtotal penetrance) in the heterozygotes and almost always total white coloring in the homozygote. This particular behavior of the mutant which we call $S^{b}$ ( $b$ for Bizet), has not yet been shown explicitely before, to our knowledge. This gene seems to exist with the same behaviour in other breeds, particularly in the Icelandic sheep. Now, when a Finnish ram, presenting the black mouflon phenotype (black with white belly), was crossed on Bizet and Solognot two allelic series were shown which control in the sheep the pigmentation itself, that is, the distribution of the two melanic pigments, black or red.

- The Extension series includes 2 alleles, one of which, $E^{d}$, is dominant over normal allele $E^{+}$. This first mutant causes epistatic generalization of black over the whole body, the Bizet would be homozygous for the dominant allele.

- The Agouti series whose action is only detectable in presence of the recessive homozygote allele of the Extension series $\left(E^{+} E^{+}\right)$; three terms seem distinguable : $A^{w h}:$ red, $A^{w}:$ black mouflon, $a$ : black ; $A^{\text {wh }}>A^{w}>a$.

The presence of the Extension series has been suggested by some authors, but no definite proof of its existence was given, up to now. The Agouti series has been already demonstrated clearly in Icelandic sheep.

The formulas of the three French breds studied may thus be written :

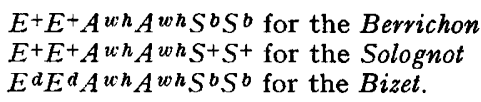

The formula of the Finnish ram could be : $E^{+} E^{+} A{ }^{w} a S^{b} S^{b}$.

Admitting that some white sheep breeds have the formula $E^{+} E^{+} A^{w h} A^{w h} S^{b} S^{b}$ (like the Berrichon), it is easily understood how the black could have been considered in the past as a recessive, not of another pigmentation mutant, but of white. In fact, $A^{w h}$ being dominant on all other terms of the Agouti series, recessive alleles may occur within a certain white breed. When they meet to produce an homozygote black aa, the gene for piebaldness changes behavior and reveals black with white points. At the contrary, in crossings with black breeds having dominant black and white points $\left(E^{d} E^{d} A^{q} A^{?} S^{b} S^{b}\right)$ the white apparently behaves as a recessive in relation to the black, $E^{d}$ being epistatic upon all the terms of the $A$ gouti series, including red $A^{\text {wh }}$.

\section{RÉFÉRENCES BIBLIOGRAPHIQUES}

Adalsternsșon S., 1960. Inheritance of coat colour in sheep. Freyr, 56, 385-39i (en islandais, résumé anglais).

Adalsteinsson S., r970. Colour inheritance in Icelandic sheep and relation between colour, fertility and fertilization. J. A gr. res. Iceland, 12, 3-1 35 .

Adalsternsson S., 1974. Colour inheritance in farm animals and its application in selection. Ist world congr. Genet. appl. Livestock Prod., 1, 29-37.

Berge S., I958. Colour in the Cld Norwegian Sheep. Meld. Norg. Landbrukshgosk., 37 (6), I-I7 et Agr. Coll. Norway Inst. Anim. Breed. Genet. Report no i rg (en norvégien, résumé anglais).

Brooker M. G., Dolling C. $\mathrm{F}^{\mathrm{r}}$.S., 1965. Pigmentation of sheep. I. Inheritance of pigmented wool in the Merino. Austr. J. agric. Res., 16, $219-228$.

Hayman R. H., Cooper D. W., 1965. The frequency of pigmented sheep in the Australian Merino. Wnol Technol. Sheep Breed., 12 (1), $8 \mathrm{I}-85$.

Hoogschages P., r967. L'hérédité de la couleur noire chez les moutons Néerlandais. Veet. Zuitelber., 10 (1), 22-27 (en hollandais).

Lauvergne J. J., 196r. Sur le déterminisme génétique de la couleur noire dans la race Bleu du Maine. Ann. Génét., 2, 47-52.

Lauvergne. J. J., r962. Évolution d'une fréquence génique dans la race ovine Bleu du Maine. Ann. Biol. anim. Bioch. Biophys., 2, I19-127.

Lauvergne J. J., 1969. Hérédité de la couleur blanche du mouton Berrichon croisé à des Solognots Ann. Génét. Sél. anim., 1, 219-226.

LAUVERGNe J. J., I97I. Mise en évidence de l'extension du phénotype noir et feu dans deux nouvelles espèces de Mammifères. Rev. roum. Biol. (sér. Zool.), 15, i $13-118$. 
Quittet E., 1965. Races ovines françaises. La Maison Rustique, Paris, 26-30, 32-33, 78-79.

RAE A. L., 1956. The genetics of the sheep. Adv. Genet., 8, 189-265.

RENDEL J., 1957. Color inheritance in farm animals. Kungl. Skogs. Landbruksakad, tidskr., 96, 208-263, (en suédois, résumé anglais).

SeARle A. G., 1968. Comparative Genetics of coat colour in Mammals. Logos Press, Academic. Press, London and New York, I89-194. 